\title{
Satisfaction survey on the critical care response team services in a teaching hospital
}

This article was published in the following Dove Press journal:

International Journal of General Medicine

I8 March 201।

Number of times this article has been viewed

\author{
Saad Al Qahtani ${ }^{1,2}$ \\ 'Intensive Care Department, Critical \\ Care Response Team, King Abdulaziz \\ Medical City (KAMC), National \\ Guard Health Affairs, ${ }^{2}$ King Saud \\ Bin Abdulaziz University for Health \\ Sciences, College of Medicine, Riyadh, \\ Kingdom of Saudi Arabia
}

Introduction: Patient care and safety is the main goal and mission of any health care provider. We surveyed nurses in the wards and obtained their feedback about the quality of care delivered by the Critical Care Response Team (CCRT).

Methods: Our hospital has 900 beds. A self-administered survey was given onsite to all ward nurses. Survey items were identified, discussed, reviewed, piloted, and finalized over a 3-month period in a focus group discussion format during three CCRT core group meetings. Responses were anonymous and collected by the nurses onsite.

Results: The total number of returned and analyzed surveys was 274 (98.6\%). Ninety-seven percent agreed that CCRT staff arrived in a timely manner. Ninety-four percent reported that CCRT staff helped in managing sick patients and $\sim 70 \%$ reported that it strengthened team dynamics. Only $50 \%$ of the nurses felt CCRT staff improved competence at the bedside. The overall satisfaction was $100 \%$; none of the nurses were dissatisfied with the team.

Conclusion: The CCRT helped manage sick patients in the wards. However, CRRT staff should remember to involve and communicate with the team initiator and the patient's physician to optimize patient health care.

Keywords: rapid response team, medical emergency team, critical care response team, satisfaction

\section{Introduction}

Implementation of a rapid response system (RRS), which is variously named a critical care response team (CCRT) in Canada, a medical emergency team (MET) in Australia, a rapid response team (RRT) in the US, and a critical care outreach team in the UK, is one of the main interventions recommended by the Institute for Healthcare Improvement Cambridge, MA in its '100,000 Lives Campaign' that was launched in 2005. This initiative is to improve the quality of care of acutely ill in-hospital patients.

The Critical Care Response Team takes the skills and expertise of the critical care team beyond the walls of the intensive care unit (ICU) within minutes, to the bedside of deteriorating patients, whose condition may well progress to cardiac or respiratory arrest. ${ }^{1}$ The CCRT stabilizes patients, prevents development of critical illness or cardiopulmonary arrest, and contributes to the optimization of the care of other patients, through education of healthcare providers working in general medical and surgical wards. Buist et al reported a reduction in unexpected deaths in hospitals from 3.77 to 2.05 per 1000 hospital admissions after implementation of MET and a decrease in cardiac arrests of from $77 \%$ to $56 \%$. $^{2}$ Bellomo et al showed reductions in 
cardiac arrests of $65 \%(P=0.001)$, deaths from cardiac arrest of $56 \%(P=0.005)$, duration of ICU stay post-arrest of $80 \%$ $(P=0.001)$, and inpatient deaths of $25 \%(P=0.004){ }^{3}$

King Abdulaziz Medical City, Riyadh (KAMC-R), Kingdom of Saudi Arabia, is a 900-bed facility including 25 inpatient medical and surgical wards. It is a tertiary care academic centre affiliated with King Saud Bin Abdulaziz University for Health Sciences, with more than 20 residency and fellowship programs. The CCRT was implemented in November 2008. The team is led by an in-house, North American board-certified intensivist, and consists of the intensivist, a staff physician, a critical care nurse, and a respiratory therapist. While there are no residents on the team, they are encouraged to attend the crises as part of the responding team. KAMC-R is a nonprofit organization, under the umbrella of the National Guard in the Kingdom of Saudi Arabia and there is no financial burden.

Prior to implementation, a CCRT policy and procedure was created and a mandatory part of this policy that the most responsible physician, or his designee, must attend the CCRT activation for maximum patient care and benefit. The patients are followed up for a minimum of 48 hours or until the crisis is resolved, or until the patient is admitted to the critical care area, pronounced dead, or a do not resuscitate order is given.

The reason for choosing the nursing staff as the target population for the survey was because they are the major end users of CCRT. Out of the total number of activations for 2 years since implementation, 67\% were activated by nursing staff, and the remainder physicians. Other than activation, the nursing staff are with the patients 24 hours a day and any CCRT follow up within the wards is with the nursing staff when they are doing patient care. Additionally, it was decided prior to CCRT implementation that activation of the team must be at the discretion of the bedside health care provider, and activation does not require a prior order by the physician.

CCRT provides services to all surgical, medical, oncology, hepatobiliary, and obstetric and gynecology adult inpatients, 24 hours a day, 7 days a week.

\section{Subjects and methods}

A self-administered survey was given on-site to all ward nurses. Approximately $70 \%$ of nurses were engaged in ward patient-care duties at the time of the survey. Nurses who were off duty at the time or on leave were excluded. Responses were anonymous.
A literature search about similar surveys was done. Items on the questionnaire were identified, discussed, reviewed, piloted, and finalized over a 3-month period in 3 CCRT core group meetings, in a focus group discussion format, which consists of 5 intensivists including the director of the team, ICU nurse manager, 3 nurse educators, clinical resource nurses, a quality improvement department representative, a respiratory therapy manager, 2 CCRT respiratory therapists, 3 CCRT nurses, a nurse coordinator (the key player of the team), and a clinical pharmacist.

Indications for CCRT activation are: change in heart rate $<60>130$ beats per minute, change in systolic blood pressure $<90>200 \mathrm{mmHg}$, change in respiratory rate $<8>30$ breaths per minute, change in saturation $<90 \%$ despite oxygen supplement, change in consciousness, a fall in Glasgow Coma Scale $<2$ points, acute change in urinary output $<50 \mathrm{~mL}$ in 4 hours, and any repeated or prolonged seizures.

The survey was formulated to evaluate the satisfaction level of the nurses with the services provided by the CCRT. It contains a total of 15 statements, 10 of which are based on a 5-point Likert scale varying from strongly disagree to strongly agree, including the following:

1. CCRT arrived in timely manner.

2. CCRT team members were respectful in their behavior and were helpful.

3. CCRT was always available when called.

4. The calling criteria were easy to understand and follow.

5. CCRT enhanced or helped improve the care of medical-surgical patients.

6. Did you feel supported by the CCRT for the patients they were following after ICU discharge?

7. CCRT promoted patient well-being by intervening at early signs of deterioration.

8. Did you feel that cardiac arrests in your ward had decreased markedly after the launching of CCRT service?

9. The in-service given by the CCRT (before and after launch) was adequate.

10. CCRT provides adequate bedside education.

11-15. About the overall performance of the CCRT.

The "strongly agree" and "agree" responses are positive responses indicating that the participants are satisfied with the team, whereas the "strongly disagree" and "disagree" responses are the negative responses indicating they are not satisfied with the team. Statements 11 to 14 are to allow the 
respondents to express their view on the CCRT and what they would like the team to know or change. Statement 15 is to rate the satisfaction level in terms of being highly satisfied, moderately satisfied, and not satisfied with all services provided by the team. Surveys were given to all nurses in the wards including nurse managers, clinical resource nurses, and bedside nurses. The survey was done after general orientation of the form through the charge nurse in the unit. It was distributed at $07 \mathrm{~h} 00$ and $19 \mathrm{~h} 00$, coinciding with shift changes in order to guarantee maximum participation. Participation was voluntary and privacy was protected since no personal information was collected such as names or any other identifying information. The charge nurse (not part of the CCRT team) collected the completed surveys and handed them to the CCRT nurse coordinator. There was no assessment of the forms or direct questioning of the nurses on the wards. There were no incentives involved for participating in the survey and participation of the nurses' was entirely voluntary. The final number of forms collected was counted and assessed by the CCRT nurse and not the ward charge nurse, to avoid any pressure on the nurse to force them to participate.

The nurses' responses were recorded and tabulated, and after assigning points to each response the values were calculated.

\section{Statistical analysis and results}

Data were entered into a Microsoft Excel program, and then transferred into Statistical Package for the Social Sciences (SPSS) (version 18) for data management and analyses. Categorical variables were summarized by calculating the number and percentage. The total number of nursing staff was $461(100 \%)$, staff on vacation $23(5 \%)$, nurses off duty 160 (35\%), and remaining participants 278 (60\%); 92.4\% were ward bedside nurses and $64.5 \%$ were from the medical units. A total of 274 (98.6\%) questionnaires was returned and analyzed.

The CCRT average response time was 6 minutes to all units, with no significant difference. There was $97 \%$ agreement that the CCRT members arrived in a timely manner and $98 \%$ agreement that the calling criteria were easy to understand and follow. Less than $2 \%$ neither agreed nor disagreed that CCRT helped in enhancing the care of surgical and medical patients. Of the participants, 99\% strongly agreed and agreed that CCRT promotes patient well-being by preventing and detecting early signs of deterioration; $24.4 \%$ felt that the CCRT did not provide adequate bedside education. Also, $7.6 \%$ of the nurses in the wards felt that the CCRT in-services were not informative and inadequate.

\section{Discussion}

Current information indicates that KAMC-R is the only intensivist-led CCRT in the Kingdom of Saudi Arabia and all neighbouring countries bringing the critical care expertise to the bedside of the patient, valuing the need for an ICU 'without-walls' concept. This project is an example of quality of care initiatives in our organization. Another survey will be conducted to measure the perception about the team's performance of other users, such as the most responsible physicians, families, and medical and nursing management. Preliminary data showed a significant reduction in the number of non-ICU cardiac arrests and unanticipated deaths, a decrease in the rate of unanticipated ICU admissions, and, ultimately, a decrease in hospital mortality after implementing the CCRT. Also, for those patients admitted to ICU, the CCRT helped to decrease the acuity of illness severity and prevent the end result multi-organ failure, hence achieving a significant reduction in ICU mortality, as previous studies have also shown. ${ }^{1}$

In a similar study, Galhotra et al suggested that most nurses surveyed had a favourable opinion of the medical emergency team, and it was suggested that a medical emergency team program be implemented as a way of improving patient care and the nurse working environment. ${ }^{4}$ Another study clearly stated that the major barrier to calling the MET appears to be allegiance to the traditional approach of initially calling parent medical unit doctors, rather than fear of being criticised for calling the MET service. ${ }^{5,6}$

The high response rate might be related to the dedicated team; the nurses who participated, the team who distributed the survey and collected them promptly without delay, and the exceptional support from the nursing higher management to make it happen. The only incentive they received was an acknowledgment from the hospital CEO and the Director of Nursing Services in the Annual Awareness CCRT week. It is believed that continuous education is imperative to strengthen the role of the team, both for afferent (care provider) and efferent limbs (CCRT). Another study concluded similarly that ongoing education on all aspects of the MET system is recommended for nursing, medical, and MET staff. Bringing MET education into undergraduate programs in order to prepare new graduates entering the workforce to care for acutely ill patients is also strongly recommended. ${ }^{7} \mathrm{KAMC}-\mathrm{R}$ is a tertiary teaching hospital with 
international staff comprising about 25 different nationalities. The considerable expansion of the work of the hospital brings new staff every year into every unit, including physicians and residents. To cover these frequently changing teams, regular education and orientation with reminders are very important for the proper utilization of this team. The CCRT's role is strengthened only if it is used to its full potential and in the right way. To support this, the role of CCRT is included in the initial general hospital orientation and then re-orientation on the wards every 3 months, and also for physicians and residents at the start of their role in the hospital and every 6 months during their departmental meetings. It has been clearly shown that education delivered to health care providers including nurses and physicians is a vital goal when implementing this team. As indicated by other studies, junior doctors and nurses should be encouraged to attend ward MET calls to gain skills in management of acutely ill patients. ${ }^{7}$ An expert opinion in the shortest possible time, on a consistent basis at the bedside, without going through the hassles of chain of command, is an achievement in itself.

Several models of leading the team have been reported, such as a nurse-led team, doctor-led team, team with a respiratory therapist, and a nurse-only team. It was decided that the team model we selected was best suited for KAMC-R patients, to bring the best care to the bedside quickly, especially since the ICU is a closed unit and the inhouse intensivist coverage is 24 hours a day, 7 days a week. Moreover, the whole concept of CCRT was to get intensivist and ICU intervention at bedside within the shortest period of time. Of all the existing models, an intensivist-led team guarantees this. The satisfaction survey was one way to getting feedback to improve the team and overcome any deficiencies to maximize utilization of the team.

Other methods are also being considered to analyze the effectiveness of the team, other than just mortality and cardiac arrest reduction. Nursing and physician satisfaction surveys and departmental feedback was one of the methods used. The team has been well received by the nurses due to the consistency of its performance, which was the main achievement. The fact that nurses activated the team about $65 \%$ of the time shows that the primary contact (the nurse) of the patient is comfortable about calling the team without fear of reprimand from the CCRT or the most responsible physician. By providing prompt help, the team is successful in doing what they are supposed to do.

\section{Acknowledgment}

I would like to thank Dr Arabi, ICU Chairman for his support, Dr Sajed Hussain and Ms Fong, CCRT Coordinators, and all the CCRT members for their dedication.

\section{Disclosure}

The author discloses no conflicts of interest.

\section{References}

1. Al-Qahtani S, Al-Dorzi HM. Rapid response systems in acute hospital care. Ann Thorac Med. 2010;5:1-4.

2. Buist MD, Jarmolowski E, Burton PR, et al. Recognising clinical instability in hospital patients before cardiac arrest or unplanned admission to intensive care. A pilot study in a tertiary-care hospital. Med J Aust. 1999;171(1):22-25.

3. Bellomo R, Goldsmith D, Uchino S, Buckmaster J, Hart GK, Opdam H, et al. A prospective before-and-after trial of a medical emergency team. Med J Aust. 2003;179:283-287.

4. Galhotra S, Scholle CC, Dew MA, Mininni NC, Clermont G, DeVita MA. Medical emergency teams: a strategy for improving patient care and nursing work environments. $J A d v$ Nurs. 2006;55:180-187.

5. Jones D, Baldwin I, McIntyre T, et al. Nurses' attitudes to a medical emergency team service in a teaching hospital. Qual Saf Health Care. 2006; 15:427-432.

6. Bagshaw SM, Mondor EE, Scouten C, et al. A survey of nurses' beliefs about the medical emergency team system in a canadian tertiary hospital. Am J Crit Care. 2010;19:74-83.

7. Jones L, King L, Wilson C. A literature review: factors that impact on nurses' effective use of the Medical Emergency Team (MET). J Clin Nurs. 2009;18:3379-3390.
International Journal of General Medicine

\section{Publish your work in this journal}

The International Journal of General Medicine is an international, peer-reviewed open-access journal that focuses on general and internal medicine, pathogenesis, epidemiology, diagnosis, monitoring and treatment protocols. The journal is characterized by the rapid reporting of reviews, original research and clinical studies across all disease areas.

\section{Dovepress}

A key focus is the elucidation of disease processes and management protocols resulting in improved outcomes for the patient.The manuscript management system is completely online and includes a very quick and fair peer-review system. Visit http://www.dovepress.com/ testimonials.php to read real quotes from published authors. 